PROCEEDINGS OF THE

AMERICAN MATHEMATICAL SOCIETY

Volume 140, Number 1, January 2012, Pages 267-278

S 0002-9939(2011)10923-7

Article electronically published on May 26, 2011

\title{
EXTREMAL KLEINIAN GROUPS
}

\author{
WILLIAM ABIKOFF AND WILLIAM J. HARVEY
}

(Communicated by Michael Wolf)

\begin{abstract}
In 1967, Lipman Bers proved his area inequalities for Kleinian groups and gave examples to show that they are sharp; a group for which equality holds is termed extremal. Maskit's work on function groups published during the next decade contained implicitly a characterization of all extremal groups for the second inequality.

Here we determine the class of extremal groups for the first area inequality: these maximal area groups are all torsion-free Schottky or almost Schottky groups. For completeness, we also show that any extremal group for the second area inequality is either quasi-Fuchsian or a regular b-group.
\end{abstract}

The purpose of this paper is to finish off a question about Kleinian groups left unresolved from the work of Ahlfors and Bers in the 1960s.

Here is a brief description of the history and the questions we answer. Some geometric estimates are implicit in Ahlfors's proof of his Finiteness Theorem [2], but his immediate goal was to apply analytic methods to study automorphic forms for the (discontinuous) action in the plane and prove finite type for the quotient Riemann surfaces which result. A gap in his argument, first noticed by Bers, was filled by Greenberg (see [6]) and, almost simultaneously, by Bers himself [5]. Bers [4] expanded his argument to produce two area inequalities for any finitely generated Kleinian group $G$ acting discretely on the plane. The first inequality was given in terms of the number of generators of $G$ : to the best of our knowledge, all basic estimates in terms of the number of generators for the geometry of a Kleinian group are linear in the number of generators. The second inequality applies to the restricted class of groups, called function groups, for which the discontinuity set has a $G$-invariant component; the structural properties underlying this inequality emerged in subsequent work of Maskit (see [14 and [13]). Bers had shown by examples that both area inequalities are sharp; later, R. Sibner asked whether the Bers examples are the only groups for which equality holds.

\section{BACKGROUND}

Let $G$ be a finitely generated Kleinian group, a discrete subgroup of the complex

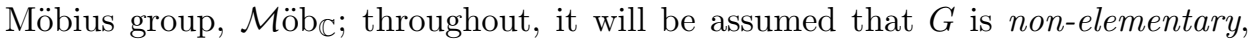
that is, not virtually abelian. Then $G$ acts conformally on the Riemann sphere $\hat{\mathbb{C}}$ and, using Poincaré's extension, by conformal isometries on the unit 3-ball $\mathcal{B} \subset \mathbb{R}^{3}$; $\hat{\mathbb{C}}$ may be viewed as the boundary (or sphere at infinity) of $\mathcal{H}^{3}$, as the ball $\mathcal{B}$ is a

Received by the editors May 14, 2010 and, in revised form, November 13, 2010.

2010 Mathematics Subject Classification. Primary 30F40; Secondary $20 \mathrm{H} 15$.

(C)2011 American Mathematical Society

Reverts to public domain 28 years from publication 
model of hyperbolic 3-space $\mathcal{H}^{3}$ and $\mathcal{M} \boldsymbol{M}_{\mathbb{C}}$ is the group of orientation-preserving isometries of $\mathcal{H}^{3}$. Let $\Omega=\Omega(G)$ be the region of discontinuity of $G$, the maximal (necessarily open) subset of $\widehat{\mathbb{C}}$ on which $G$ acts properly discontinuously. In all cases of interest to us here, $\Omega \neq \emptyset$; in some circles one says that such a $G$ is Kleinian of the second kind.

If $G$ has no torsion, $X(G):=\left[\mathcal{H}^{3} \cup \Omega\right] / G$ is a bordered 3-manifold whose interior carries a complete hyperbolic structure. In the torsion case, it is a bordered 3orbifold whose interior has a complete hyperbolic cone structure. The quotient 2-manifold or orbifold $\Omega / G$ is called the conformal boundary of $X(G)$.

When a group $G$ acts on a space $Y$, a subset $A \subset Y$ is called precisely invariant under the subgroup $H<G$ if $H A \subset A$ and $\gamma(A) \cap A=\emptyset$ whenever $\gamma \in G-H$. In this situation we also refer to $H$ as the isotropy or stability subgroup of $A$ and denote it $G_{A}$.

For the reader's convenience, we discuss briefly the types of groups which are relevant to this study. A Kleinian group $G$ is called a function group if there is a connected component $\Omega_{0}(G)$ of $\Omega(G)$ which is invariant under the action of $G$. This class contains a subclass of particular interest to us, the Schottky groups; they are defined as follows. Start with a collection $\left\{\left(C_{i}, C_{i}^{\prime}\right) \mid i=1, \ldots, g\right\}$ of pairwise disjoint Jordan curves in $\widehat{\mathbb{C}}$ which has the property that no two curves are separated by a third. Assume that there are Möbius transformations $\gamma_{i}, i=1, \ldots, g$ so that $\gamma_{i}\left(\operatorname{Ext}\left(C_{i}\right)\right)=\operatorname{Int}\left(C_{i}^{\prime}\right)$ for each $i$ and $G$ is the group generated by the $\gamma_{i}$. Then $G$ is freely generated by the $\gamma_{i}$, is purely loxodromic (i.e. each $\gamma \in G-\{$ id $\}$ is loxodromic) and $X(G)$ is a handlebody of genus $g$; the discontinuity set $\Omega(G)$ is connected.

A group $G$ is said to be of Schottky type if there is a Schottky group $H$ and an isomorphism $h: H \rightarrow G$ with the following properties:

(1) $h$ is induced by a continuous surjection $\eta: \hat{\mathbb{C}} \rightarrow \widehat{\mathbb{C}}$ satisfying $\eta G=H \eta$.

(2) for any point $z \in \hat{\mathbb{C}}$, either $\eta^{-1}(z)$ is a point or $z$ is the fixed point of a parabolic element $\gamma \in G$ and $\alpha:=\eta^{-1}(z)$ is a simple arc invariant under $h^{-1}(\gamma)$

(3) Curves $\alpha$ corresponding to distinct elements $\gamma \in G$ are either identical or may be chosen to be disjoint.

The groups of Schottky type are best understood in terms of their quotient surfaces $S_{G}:=\Omega(G) / G$. As a topological space, $S_{G}$ may be obtained from the closed surface $S_{H}$ by collapsing some collection of disjoint closed simple geodesics to points; the points are often called cusps. We will show later that this topological collapsing operation is reversible in a natural way.

A finitely generated function group $G$ is called quasi-Fuchsian if $\Omega(G)$ has two simply connected invariant components and is termed a b-group if it has precisely one.

The limit set of a Kleinian group $G$ is $\Lambda=\Lambda(G)=\hat{\mathbb{C}}-\Omega(G)$. Since $G$ is not virtually abelian, $\Lambda$ is a non-empty perfect set and consequently has at least three points. It follows that each component of $\Omega$ carries a hyperbolic metric which projects to $\Omega / G$. Thus the quotient $\Omega / G$ is a union of hyperbolic 2 -orbifolds or, in more classical terminology, hyperbolic Riemann surfaces with signature, that is, having a set of singular points, comprising cusps or cone points with cone angles $2 \pi / n$ for some integers $n \geq 2$. 
Theorem 1.1 (The Ahlfors Finiteness Theorem). If $G$ is a finitely generated Kleinian group, then $\Omega / G$ is a finite union of conformally finite hyperbolic 2orbifolds (i.e. Riemann surfaces with signature having finite hyperbolic area).

1.1. Geometric finiteness. The property of geometric finiteness arose first in the aftermath of the finiteness theorem, with Greenberg's discovery that the important subclass of so-called degenerate b-groups discovered by Bers and Maskit could not have finite-sided fundamental polyhedra in $\mathcal{H}^{3}$, thus providing a clear distinction from the two-dimensional case of Fuchsian groups acting on the hyperbolic plane. Since then, this has become a topic of great interest for Kleinian groups and, more generally, for geometric group theory.

There are many equivalent conditions under which we say that a Kleinian group $G$ is geometrically finite. Here are two of them; for others, and for a detailed discussion, the reader may consult [7].

(1) (Ahlfors) Assume $w_{0} \in \mathcal{H}^{3}$ is not an elliptic fixed point and $d$ denotes hyperbolic distance in $\mathcal{H}^{3}$. The Dirichlet region for $G$ based at $w_{0}$ is defined by

$$
D\left(G, w_{0}\right):=\bigcap_{\gamma \in G-\{\mathrm{id}\}}\left\{w \in \mathcal{H}^{3} \mid d\left(w, w_{0}\right)<d\left(w, \gamma\left(w_{0}\right)\right\} .\right.
$$

$G$ is geometrically finite if $D\left(G, w_{0}\right)$ has a finite number of sides. Note that the condition does not depend on the choice of basepoint.

(2) (Thurston) Let $\mathcal{O}=\mathcal{H}^{3} / G$. The Nielsen core $K(G)$ of $G$ is the smallest closed, convex set in $\mathcal{H}^{3}$ which contains all hyperbolic lines connecting any two limit points. $G$ is geometrically finite if the convex core $C(G):=K(G) / G$ has finite hyperbolic volume. Note that this characterization requires the separate assumption that $G$ is finitely generated.

Abikoff [1] showed, using results of Maskit [12, Beardon and Maskit [3] and Marden [10], that every geometrically finite function group with $\Omega_{0}$ simply connected is either quasi-Fuchsian or a regular b-group. The topological structure of a regular b-group is obtained from that of a quasi-Fuchsian group by pinching to points a non-empty collection consisting of disjoint (i) closed geodesics and/or (ii) simple geodesic arcs connecting branch points of order 2 on a single component of $\Omega / G$. The metric is the hyperbolic metric on that component.

1.2. The Euler characteristic and hyperbolic area. The Euler characteristic $\chi(C)$ of a finite simplicial complex is the usual Euler characteristic and may be computed as an alternating sum of the number of cells in a given dimension. It is zero for closed odd-dimensional manifolds and, for a closed orientable surface $S$ of genus $g,-\chi(S)=2(g-1)$. For manifolds, in contrast to orbifolds, it is the alternating sum of the Betti numbers $\beta_{i}$.

Orbifolds of dimension two and three have an underlying topological structure of a manifold of the same dimension, but the Euler characteristic of that structure is not sensitive to the original orbifold structure. C. T. C. Wall 18 introduced the more general notion of virtual Euler characteristic and showed that it is well-defined for orbifolds and natural under finite degree coverings. The account of virtual Euler characteristics which follows is restricted to the results we need later.

It is convenient for us to set $\xi(X)=-\chi(X)$ both for the usual and virtual Euler characteristics. 
Given an orbifold $\mathcal{O}$ which is realized as $M / \Gamma$ with $M$ a compact, bordered differentiable manifold, necessarily of the same dimension as $\mathcal{O}$, and $\Gamma$ a finite group of diffeomorphisms of $M$ with order $|\Gamma|=N$, we define $\xi(\mathcal{O}):=\xi(M) / N$; $\xi$ then takes on rational values. It is known that $\xi(\mathcal{O})$ is well-defined and is determined by the orbifold universal cover group of $\mathcal{O}$. In our situation, $\mathcal{O}$ has dimension 2 or 3 and the universal cover $\mathcal{H}^{n}$ has a natural metric structure invariant under the group of hyperbolic isometries.

It follows from the vanishing of $\xi$ for odd-dimensional closed manifolds that the Euler characteristic of a compact, bordered, odd-dimensional manifold is twice the Euler characteristic of its border. The same result is true for odd-dimensional orbifolds $X$, i.e.

$$
\xi(\partial X)=2 \xi(X) .
$$

Let $X$ be an orientable 2-orbifold which admits a finite sheeted covering by a hyperbolic surface $S$. The classical Riemann-Hurwitz Theorem compares the two Euler characteristics for this branched covering of Riemann surfaces. We assume that $S$ (and therefore $X$ also) is compact with corresponding genera $g_{S}$ and $g_{X}$ respectively. Further, let $p_{1}, \ldots, p_{i}, \ldots, p_{m}$ be a list of the branch points of order $\nu_{i}$, respectively; equivalently, they are the cone points on $X$ with cone angle $2 \pi / \nu_{i}$. The total branching number of the covering is

$$
B:=\sum_{i=1}^{m}\left(\nu_{i}-1\right) \text {. }
$$

The theorem, for a branched covering of surfaces, states that

$$
g_{S}-1=n\left(g_{X}-1\right)+B / 2 .
$$

The proof is by a counting argument using fundamental domains. One computes the hyperbolic area of $S$ from the area of $X$ as follows: use the above notation and let $z_{m+1}, \ldots, z_{k}$ be the cusps (or punctures) of $X$. We treat a puncture as a branch point of order $\infty$ (or cone angle zero). Then, from the Gauss-Bonnet theorem, the hyperbolic area of $X$ is

$$
\begin{aligned}
A(X) & =2 \pi \cdot\left(2 g-2+\sum_{i=1}^{n}\left(1-\frac{1}{\nu_{i}}\right)\right) \\
& =2 \pi \xi(X) .
\end{aligned}
$$

The Riemann-Hurwitz formula is equivalent to the statement $\xi(S)=n \xi(X)$ or, in other words, that the orbifold Euler characteristic is multiplicative on branched coverings. In this formulation, it is easily seen to hold for all finite branched coverings of hyperbolic 2-orbifolds with finite area.

Here is a list of standard facts that we will need later:

(1) The area of a disjoint union of connected 2-orbifolds is (defined to be) the sum of the areas of the components.

(2) If $X$ is a hyperbolic 2-orbifold and $\alpha$ is a simple closed geodesic on $X$ which does not intersect the cone locus, then we can pinch $\alpha$ to a point and remove that point. The resulting topological surface has either one or two components depending on whether $\alpha$ is non-dividing or not. If each orbifold component is given a hyperbolic metric, the pinching operation 
does not change the total area, because two fresh punctures result from each pinching.

(3) Let $\beta$ be a simple geodesic arc on $X$ which connects two branch points of order two. Then $\beta$ may be collapsed to a point $p$ having a non-standard cone structure. Any point $\tilde{p}$ covering $p$ becomes a puncture whose isotropy group is infinite dihedral with a parabolic subgroup of index 2. Again there is no change in the area of $X$ when $\beta$ is collapsed to a point considered as a single puncture.

It follows from the two previous statements that

(4) a regular b-group $G$ is a geometrically finite function group with a single invariant component $\Omega_{0}$ which is simply connected; the area of the conformal boundary of $X(G)$ satisfies $A(\Omega / G)=2 A\left(\Omega_{0} / G\right)$.

1.3. The Bers area inequalities. The rank of a Kleinian group $G$, denoted $r(G)$, is the minimal number of generators of $G$. $G$ is called a maximal area (or extremal) group if, among all groups of rank equal to $r(G), \Omega(G) / G$ has maximal hyperbolic area. The first of the area inequalities shows that there is a rank-dependent bound on area and that maximal area groups exist.

Theorem 1.2 (Bers' First Area Inequality). Suppose $G$ is a Kleinian group with $\operatorname{rank} r(G)=N$. Then

$$
A(\Omega / G) \leq 4 \pi(N-1) .
$$

Equality holds for any Schottky group.

Equality holds in this equation if and only if $G$ is a maximal area or extremal group.

We include, for completeness, the statement of the Second Inequality and later give a characterization of its extremal groups.

Theorem 1.3. (Bers' Second Area Inequality) Suppose $G$ is a finitely generated function group with invariant component $\Omega_{0}$. Then

$$
A(\Omega / G) \leq 2 A\left(\Omega_{0} / G\right) .
$$

Equality holds for any Fuchsian group.

1.4. Coverings by manifolds and the corresponding group theory. We continue to assume that $G$ is finitely generated. By the Selberg Lemma, $G$ has a normal subgroup $H$ of finite index $n$ without torsion. Thus $X(G)$ has a finite sheeted orbifold cover by a bordered manifold $X(H)$. Then $\xi(X(G))=\xi(X(H)) / n$. In general, this is quite different from $\xi(M(G))$, where $M(G)$ is the 3 -manifold underlying $X(G)$. Indeed, the relationship between the two is not well understood.

The orbifold fundamental group $\pi_{1} X(G)$ of $X(G)$ is defined to be equal to the group $G$ in the Kleinian case.

For a general orbifold there is a rather cumbersome homotopy-theoretic definition which we shall not need; see for instance [16].

Here is a useful elementary result from classical discrete group theory (see Lyndon and Schupp [8, p. 164]): it follows immediately from the theorems of J. Nielsen and O. Schreier on finite index subgroups of free groups. 
Lemma 1.4 (The Rank Inequality). Let $H$ be a subgroup of index $n$ in a finitely generated discrete group $G$. Then $H$ is finitely generated and

$$
r(H)-1 \leq n(r(G)-1) .
$$

Proposition 1.5. Suppose $G$ is a finitely generated extremal (i.e. maximal area) group and $H<G$ is a subgroup of finite index. Then $H$ is also extremal.

Proof. Let $n$ be the index of $H$ in $G$; then $X(H)$ is an $n$-sheeted orbifold cover of $X(G)$. Furthermore, $G$ and $H$ have the same discontinuity set $\Omega=\Omega(G)$. It follows that $A(\Omega / H)=n \cdot A(\Omega / G)$. Using the first Area Inequality and the Rank Inequality, we obtain

$$
r(H)-1 \geq \frac{A(\Omega / H)}{4 \pi}=\frac{n \cdot A(\Omega / G)}{4 \pi}=n(r(G)-1) \geq r(H)-1 .
$$

It follows that $H$ satisfies the first area equality; it is therefore a maximal area group.

We note that the converse is not true; that is, if $H$ has maximal area, it does not follow that $G$ does. However, topological arguments give an efficient proof of the first Area Inequality for torsion free groups: the argument is given in Section 3 , This inequality then follows immediately for all finitely generated groups from the next result.

Proposition 1.6. Suppose $G$ is a finitely generated group and $H<G$ is a subgroup of finite index $n$. If $H$ satisfies the inequality in Equation (3), then so does $G$.

Proof. We use the notation of the previous proposition:

$$
A(\Omega / G)=\frac{A(\Omega / H)}{n} \leq \frac{4 \pi \cdot(r(H)-1)}{n} \leq 4 \pi \cdot(r(G)-1) .
$$

1.5. The compact core of a hyperbolic 3-manifold and pared orbifolds. Following earlier work of G. A. Swarup, Peter Scott gave a valuable topological description of 3-manifolds with finitely generated fundamental group.

Theorem 1.7 (Scott's Core Theorem). Suppose $M$ is a 3-manifold with finitely generated fundamental group. Then there is a compact submanifold $i: N \subset M$ so that $\pi_{1}(i)$ is an isomorphism. Furthermore:

- If Int $M$ is hyperbolic, we may fill in any omitted balls so that $i$ is a homotopy equivalence.

- (McCullough [15]) If $F$ is any compact subset of $\partial M, N$ can be chosen so that $F \subset \partial N$.

Next suppose that $X=\left[\mathcal{H}^{3} \cup \Omega(G)\right] / G$ is a hyperbolic 3-orbifold together with its conformal boundary. Let $P_{\lambda}$ be a maximal parabolic subgroup stabilizing the point $\lambda$ and let $N_{\lambda}$ be its normalizer in $G$. Then there is an open Euclidean ball, called a horoball, $\mathfrak{h} \subset \mathcal{H}^{3}$, which is tangent to $\partial \mathcal{H}^{3}$ at $\lambda$ and is precisely invariant under $N_{\lambda}$. Let $E_{\lambda}^{\prime}:=\mathfrak{h} / N_{\lambda}$; there is an inclusion $i: E_{\lambda}^{\prime} \hookrightarrow X$. If $P_{\lambda}$ has rank two, $E_{\lambda}^{\prime}$ is (orbifold) homeomorphic to $\mathcal{O}_{2} \times[0, \infty)$, where $\mathcal{O}_{2}$ is an orientable, compact Euclidean 2-orbifold. If $N_{\lambda}$ contains torsion, $E^{\prime}$ is called a virtual cusp torus.

Now we assume that $P_{\lambda}$ is cyclic. There are several possibilities.

If $P_{\lambda} \subset G_{1}=G_{\Omega_{1}}$, the stabilizer in $G$ of a component $\Omega_{1}$ of $\Omega(G)$, then $\Omega_{1} / G_{1}$ is a 2-orbifold in the conformal boundary of $X$. 
First of all, if $P_{\lambda}$ is associated to a puncture on $\Omega_{1} / G_{1}$, then there is an open Euclidean 2-disk $\Delta \subset \Omega_{1}$ with $\lambda \in \partial \Delta$ which is precisely invariant under the stabilizer $\left(G_{1}\right)_{\lambda}$ of $\lambda$ in $G_{1}$. For $\Delta$ sufficiently small, it can be rotated about its own tangent line at $\lambda$ into $\mathcal{H}^{3}$ until it is contained in an invariant horoball $\mathfrak{h}$ at $\lambda$; in the process, a solid open tube $T_{1}$ is swept out in $\mathcal{H}^{3}$. If $P_{\lambda}$ lies in the stability subgroup of another component $\Omega_{2} \neq \Omega_{1}$ of $\Omega(G)$ and determines a puncture there, we obtain a second tube $T_{2}$, which we can assume to be disjoint from $T_{1}$. Then $T_{1} \cup \mathfrak{h}$ or $T_{1} \cup T_{2} \cup \mathfrak{h}$ is precisely invariant under $N_{\lambda}$ and $E_{\lambda}$ is either $\left(T_{1} \cup T_{2} \cup \mathfrak{h}\right) / N_{\lambda}$ or $\left(T_{1} \cup \mathfrak{h}\right) / N_{\lambda}$ or $\mathfrak{h} / N_{\lambda}$ (depending on whether $T_{1}$ and/or $T_{j}$ exist or neither exist) and injects into $X$. The pared orbifold $X_{\text {pared }}=(X, E)$ of $X$ is defined to be $X-E$, where $E:=\bigcup_{\lambda} E_{\lambda}$. It is unique up to (orbifold) homeomorphism compatible with the local finite group actions.

The second possibility is that the cyclic group $P_{\lambda}$ stabilizes a component $\Omega_{1}$ of $\Omega(G)$, but does not determine a puncture there. Then there is a hyperbolic geodesic $\ell \in \Omega_{1}$ which is invariant under $P_{\lambda}$, and $\ell \cup \lambda$ is a Jordan curve. In this case, any non-trivial element of $P_{\lambda}$ is called an accidental parabolic transformation (with respect to $\Omega_{1}$ ).

Each component of the pared boundary $\partial P$ is called a cusp cylinder or rank one cusp, possibly virtual depending on $N_{\lambda}$.

\section{Statement of THE THEOREMS}

Here we characterise the extremal groups for the two Bers area inequalities. First we state our main result.

Theorem 2.1. Suppose $G$ is a Kleinian group of rank $N$ which satisfies the first area equality, i.e.

$$
A(\Omega / G)=4 \pi(N-1)=2 \pi \xi(X(G)),
$$

where $\xi$ is the negative of the virtual Euler characteristic and $X(G)$ is the quotient orbifold of $G$. Then $G$ is torsion-free and of Schottky type.

Thus $G$ is obtained from a Schottky group by making $\kappa \geq 0$ curve-pairing generators become parabolic. $G$ is Schottky if and only if $\kappa=0$.

For equality in the second area inequality, the groups in question are given as follows.

Theorem 2.2. Suppose $G$ is a finitely generated function group with invariant component $\Omega_{0}$. Then

$$
A(\Omega / G) \leq 2 \cdot A\left(\Omega_{0} / G\right),
$$

with equality if and only if $G$ is either quasi-Fuchsian or a regular b-group.

\section{Proof of Theorem 2.1 when $G$ is torsion free}

Assume $G$ is a maximal area, torsion-free Kleinian group with quotient manifold $X(G)$. We first pare $X(G)$ and then let $Y$ be a compact core of the pared manifold. As noted in Section 1.5, we may assume that $S:=\partial Y$ contains any specified compact subsurface of $\partial X(G)$. In particular, we assume that $S$ contains the quotient by $G$ of $\Omega(G)-\left\{\right.$ horocyclic neighborhoods of the cusps\}. Let $\beta_{i}$ be the $i$-th Betti number of $Y$. Then, by Equation (2),

$$
A(S / G)=2 \pi \xi(\partial Y)=4 \pi \xi(Y)
$$


Since $\beta_{1} \leq r(G)$, we have

$$
A(S) \leq 4 \pi\left[r(G)-1-\beta_{2}\right] .
$$

From $\beta_{2} \geq 0$, we obtain

$$
A(S) \leq 4 \pi[r(G)-1],
$$

which is the first area inequality for groups without torsion.

Because $G$ has maximal area, $\beta_{2}=0$ and $r(G)=\beta_{1}$. It follows that $\partial Y$ is connected, since otherwise there would be a component of $\partial Y$ that did not bound and $\beta_{2}$ would then be positive. Hence $G$ has no rank 2 cusps. Also note that since $\beta_{2}=0$, there are no degenerated components in $\partial Y$ and so $G$ is geometrically finite.

Suppose we have a rank one parabolic subgroup in $G$, generated by a transformation $\gamma$. Then there is a simple loop $\alpha \subset S$ corresponding to $\gamma$. To comprehend the action of $\gamma$ on $\mathcal{H}^{3} \cup \Omega(G)$, the fixed point $\lambda$ of $\gamma$ may be split into two points: $\lambda_{+}$(respectively, $\lambda_{-}$) which is the limit of $\gamma^{n}(w)$ (respectively, of $\gamma^{-n}(w)$ ) for any $w \in \overline{\mathcal{H}^{3}}-\{\lambda\}$. This operation is called opening a cusp: $\gamma$ is replaced by a transformation $\eta$ of infinite order and having two fixed points; that is, $\eta$ acts topologically on the universal cover of the pared 3-manifold as if it were loxodromic. The replacement of all rank one parabolic generators by topologically loxodromic transformations results in a group $H$, topologically conjugate (hence isomorphic) to $G$, which acts properly and discretely on a closed 3-ball.

By applying Thurston's hyperbolization theorem for Haken manifolds to $Y$ (see Kapovich [7]), one can, in fact, give $Y$ a hyperbolic structure so that $H$ is (conjugate to) a Kleinian group of deck transformations, but the present argument (this was pointed out to us by the referee) can be made essentially topological. Since $Y$ has no rank 2 parabolics, $H$ is purely loxodromic and also satisfies the first area equality. Therefore the pared boundary $S=\Omega(H) / H$ is a connected surface of genus $N:=r(G)$. It then follows from Teichmüller's theorem that the complex dimension of the deformation space of $S$, and also that of $H$, is $3 N-3$ : these spaces have equal dimension by Sullivan's rigidity theorem [17.

Now the deformation space of an $N$-generator Kleinian group is parametrized by a suitable finite set of traces of words in the generating set; hence, after some normalization, it has a structure of a closed subvariety $D(H)$ in a complex affine $(3 N-3)$-dimensional space $V$. Any relation in $H$ would induce a polynomial relation holding on $D(H)$, and so the deformation variety would have positive codimension in $V$. It follows that $H$, and hence also $G$, is a free group. By Maskit's characterization of Schottky groups [11, a free, purely loxodromic Kleinian group with $\Omega \neq \emptyset$ is Schottky. Therefore $C(G)$ is a handlebody and $M(G)$ is obtained from a handlebody by pinching a finite set of loops on $\partial M(G)$. Thus $G$ is of Schottky-type; it is Schottky if there are no rank 1 parabolics.

The proof of the torsion-free case is complete.

We complete the proof of Theorem 2.1] in the next section.

\section{Maximal area Groups are torsion-Free}

Assume now that $G$ is extremal (that is, a finitely generated Kleinian group of maximal area) containing torsion and let $H \triangleleft G$ be a torsion-free normal subgroup of index $n<\infty$. We have already shown that $H$ is also extremal and (in the last section) that $H$ is either Schottky or Schottky-type. In particular, $H$ has no degenerate ends; hence $G$ shares this property. As in the previous section, we may 
open all the cusps in $H$, making it Schottky. In particular, $H$ has no cusped tori, so it follows immediately that $G$ has no virtual cusped tori.

Since $\Omega(G)=\Omega(H)$, the mapping $f: S_{1}=M(H) \rightarrow M(G)=S$ is a possibly branched $n$-sheeted covering. Let $g_{1}$ be the genus of $S_{1}, g$ the genus of $S$ and $B$ the total branch number of the covering. Since $H$ is extremal and Schottky,

$$
g_{1}-1=n(r(G)-1)
$$

By the Riemann-Hurwitz formula we have

$$
\left(g_{1}-1\right)=n(g-1)+B / 2 .
$$

Also, the rank inequality, together with the extremality of $G$ and $H$, implies that

$$
\begin{aligned}
A\left(S_{1}\right) & =4 \pi\left(g_{1}-1\right)=n \cdot A(S) \\
& =2 \pi n\left(2 g-2+\sum\left(1-\frac{1}{\nu_{j}}\right)\right),
\end{aligned}
$$

where the $\nu_{j}$ are all the indices of ramification at the branch points on $S$. By combining these two equations we obtain

$$
n(g-1)+\frac{1}{2} \sum\left(1-\frac{1}{\nu_{j}}\right)=n(g-1)+\frac{B}{2} .
$$

Since $B=\sum\left(\nu_{j}-1\right)$, we infer that

$$
\sum \frac{\nu_{j}-1}{\nu_{j}}=\frac{1}{n} \sum\left(\nu_{j}-1\right) .
$$

It follows that

$$
\sum\left(\nu_{j}-1\right)\left(\frac{n-\nu_{j}}{n \nu_{j}}\right)=0 .
$$

The number of ramification points is finite and each of the $\nu_{j}>1$; also $n=[G: H]$ may be chosen arbitrarily large. We have found positive numbers whose sum is zero, so the sum must have an empty index set, and hence the covering of $S$ by $S_{1}$ is unramified.

Then, by the extremality of $G$ and $H$,

$$
4 \pi(r(H)-1)=A\left(S_{1}\right)=n A(S)=4 \pi n(r(G)-1)
$$

and

$$
2 \pi\left(2 g_{1}-2\right)=A\left(S_{1}\right)=n A(S)=2 \pi n(2 g-2) .
$$

It follows that the rank of $G$ is $g$. Repeating the argument given earlier in the torsion-free case, the deformation space $T(S)$ of $S$ is an analytic subvariety of $T(G)$ of full dimension $3 g-3$. Hence there are no non-trivial relations in $G$, so $G$ is torsion-free and the proof is complete.

It is also possible to prove this fact via Bass-Serre theory, using the induced action of a (torsion) virtually Schottky group $G$ on the Cayley graph of the Schottky subgroup $H$. 


\section{Extremal function groups for Bers' SECOND AREA InEQuality}

A finitely generated function group $G$ with invariant component $\Omega_{0}$ is called extremal for the second area inequality if

$$
A(\Omega / G)=2 A\left(\Omega_{0} / G\right) .
$$

The characterization of extremal function groups is implicitly contained in Maskit's comprehensive work on function groups (see e.g. [14 or [13]). Here we offer a proof that is perhaps less formidable. The precise statement is as follows.

Theorem 5.1. Suppose $G$ is a finitely generated function group. Then the following are equivalent:

(1) $G$ is extremal.

(2) $G$ is either a quasi-Fuchsian group of the first kind or a regular b-group.

(3) $G$ is a geometrically finite function group with simply connected invariant component.

Proof. Let $\Omega / G=\bigsqcup_{i=0}^{k} \Omega_{i} / G_{i}$, where $G_{i}$ is the $G$-stabilizer of the component $\Omega_{i}$ of $\Omega(G)$. Let $\Omega_{0}$ be the invariant component, so that $G_{0}=G$. The equivalence of (2) and (3) was given in Abikoff [1, but, with little effort, it follows from earlier work of Marden ([10] and [9]) on the torsion-free case.

If $G$ is a quasi-Fuchsian group of the first kind, then there is an orientationreversing quasiconformal map from $\Omega_{0} / G$ to $\Omega_{1} / G_{1}$, and these two components form the conformal boundary of the 3-orbifold of $G$ and have the same area. As noted previously, a regular b-group is obtained from a quasi-Fuchsian group by collapsing a collection of disjoint geodesics, each invariant under a cyclic group of loxodromic transformations to points invariant under parabolic transformations: the collapsed geodesics lie in a single component of the quasi-Fuchsian group. This does not alter the area of the quotient. Therefore quasi-Fuchsian groups and regular b-groups are extremal.

It remains to prove that the first condition implies one of the others. Suppose that $G$ is extremal and $H$ is a subgroup of index $n ; H$ is also a function group. Since $\Omega(H)=\Omega(G)$ and the area of a $k$-sheeted covering is $k$ times the area of the base, extremality is ancestral; i.e., a group is extremal if and only if all its finite index subgroups are extremal. Again, using the Selberg Lemma, we may assume $G$ is torsion-free.

Let $S_{0}=\Omega_{0}(G), S=\Omega(G) / G$ and $S_{1}=S-S_{0}$. We cannot assume $S_{1}$ is connected.

We claim that $G$ is geometrically finite. If not, there is a degenerate end $E$ of $X(G)$ which does not come from a degeneration of the invariant component. We truncate the degenerate end and obtain a hyperbolic structure on the resulting submanifold $Y$ which is compact except for cusps. One method to obtain $Y$ copies is the technique used in proving Theorem 2.1 first pare $X(G)$, then take a compact core. Attach the doubly cusped ends and shrink to points any geodesics on the boundary that correspond to cusp cylinders in $X(G)$. We have assumed that $G$ is extremal, so $A\left(S_{1}\right)=A\left(S_{0}\right)$. Further, by the second area inequality, no torsionfree function group $H$ with $\Omega_{0}(H) / H$ homeomorphic to $S_{0}$ can have $A((\Omega(H)-$ $\left.\left.\Omega_{0}(H)\right) / H\right)>A\left(S_{0}\right)$. Let $H$ be a Kleinian group whose quotient manifold is $Y$. Then $S_{0}=\Omega_{0}(H) / H$, but $\left(\Omega(H)-\Omega_{0}(H)\right) / H$ properly contains a surface quasiconformal to $S_{1}$. This contradicts the second area inequality. So we may 
assume that $G$ is geometrically finite, torsion-free, $\Omega_{0}$ is not simply connected, and $G$ is neither quasi-Fuchsian nor a regular b-group.

Any two components of $\hat{\mathbb{C}}-\Omega_{0}$ may be separated by a Jordan curve in $\Omega_{0}$, hence by a closed geodesic $\tilde{\alpha}$ there. As a Jordan curve on the unit 2 -sphere, $\tilde{\alpha}$ bounds a disk in the closed 3-ball and hence projects to a non-trivial simple closed geodesic $\alpha$ on $S_{0}$, nullhomotopic in $X=X(G)$. The covering projection $p: \tilde{\alpha} \rightarrow \alpha$ is finite sheeted; hence $\pi$ is conjugate to the map $z \mapsto z^{k}$ on the unit circle in $\mathbb{C}$ for some $k \geq 2$. We may choose a disk $D \subset \mathcal{H}^{3}$ so that $\partial D=\tilde{\alpha}$ and $D$ is invariant under the projection to $X(G)$ : such a disk is obtained as a component of the boundary of the convex hull of $\tilde{\alpha}$ in $\mathcal{H}^{3}$. G contains a transformation $\gamma$ which is of finite order and leaves $D$ invariant. By the Brouwer fixed point theorem, $\gamma$ has a fixed point in $D$ which projects to a branch point of order $k$ on $\pi(D)$.

Now we estimate areas: $A\left(S(G)-S_{0}(G)\right) \leq A\left(S_{0}(G)\right)$. When we cut $X(G)$ along $D^{\prime}:=\pi(D), S-S_{0}$ remains unchanged; hence so does its virtual Euler characteristic. However the virtual Euler characteristic of $S_{0}$ is increased by $2 / k$. To see this, note that the cutting operation either reduces the genus of $S_{0}$ by one or cuts $S_{0}$ into two pieces; at the same time we introduce two branch points of order $k$. The dissected 3-orbifold has one or two components; in each case it has a hyperbolic structure. In both cases, we obtain a function group violating the second area theorem.

\section{REFERENCES}

1. William Abikoff, On boundaries of Teichmüller spaces and on Kleinian groups. III, Acta Math. 134 (1975), 211-237. MR0435452 (55:8412)

2. Lars V. Ahlfors, Finitely generated Kleinian groups, Amer. J. Math. 86 (1964), 413-429. MR 0167618 (29:4890)

3. Alan F. Beardon and Bernard Maskit, Limit points of Kleinian groups and finite sided fundamental polyhedra, Acta Math. 132 (1974), 1-12. MR0333164(48:11489)

4. Lipman Bers, Inequalities for finitely generated Kleinian groups, J. Analyse Math. 18 (1967), 23-41. MR0229817 (37:5383)

5. _ On Ahlfors' finiteness theorem, Amer. J. Math. 89 (1967), 1078-1082. MR0222282 (36:5334)

6. L. Greenberg, On a theorem of Ahlfors and conjugate subgroups of Kleinian groups, Amer. J. Math. 89 (1967), 56-68. MR0209471 (35:369)

7. Michael Kapovich, Hyperbolic manifolds and discrete groups, Progress in Mathematics, vol. 183, Birkhäuser Boston Inc., Boston, MA, 2001. MR1792613 (2002m:57018)

8. Roger C. Lyndon and Paul E. Schupp, Combinatorial group theory, Springer-Verlag, Berlin, 1977, Ergebnisse der Mathematik und ihrer Grenzgebiete, Band 89. MR 0577064 (58:28182)

9. Albert Marden, On Bers' boundary of Teichmüller space, abstract, Notices of the Amer. Math. Soc. 18 (1971), 1082.

10. _ The geometry of finitely generated Kleinian groups, Ann. of Math. (2) 99 (1974), 383-462. MR0349992 (50:2485)

11. Bernard Maskit, A characterization of Schottky groups, J. Analyse Math. 19 (1967), 227-230. MR 0220929 (36:3981)

12. 91 (1970), 607-639. MR0297993 (45:7045)

13. _ Decomposition of certain Kleinian groups, Acta Math. 130 (1973), 243-263. MR0404614 (53:8414)

14. Kleinian groups, Springer-Verlag, 1988. MR959135(90a:30132)

15. Darryl McCullough, Compact submanifolds of 3-manifolds with boundary, Quart. J. Math. Oxford Ser. (2) 37 (1986), no. 147, 299-307. MR854628 (88d:57012)

16. John G. Ratcliffe, Foundations of hyperbolic manifolds, second ed., Graduate Texts in Mathematics, vol. 149, Springer, New York, 2006. MR2249478 (2007d:57029) 
17. Dennis Sullivan, On the ergodic theory at infinity of an arbitrary discrete group of hyperbolic motions, Riemann surfaces and related topics: Proceedings of the 1978 Stony Brook Conference (State Univ. New York, Stony Brook, N.Y., 1978), Ann. of Math. Stud., vol. 97, Princeton Univ. Press, 1981, pp. 465-496. MR624833 (83f:58052)

18. C. T. C. Wall, Rational Euler characteristics, Proc. Cambridge Philos. Soc. 57 (1961), 182184. MR0122853(23:A185)

Department of Mathematics, University of Connecticut at Storrs, Storrs, ConNECTICUT 06269-3009

E-mail address: abikoff@math.uconn.edu

Department of Mathematics, King's College, Strand, London, WC2R-2LS England

E-mail address: Bill.Harvey@kcl.ac.uk 